\title{
INFLUENCIA DE LA PÉRDIDA FOLIAR SOBRE LA COSECHA EN EL CV. GRUESA, Musa Acuminata COLLA (AAA), CULTIVADO BAJO INVERNADERO EN LAS ISLAS CANARIAS ${ }^{1}$
}

\author{
JUAN CABRERA CABRERA², ITZIAR RODRÍGUEZ HERGUETA, VÍCTOR GALÁN SAÚCO4
}

RESUMEN- El cultivo de la variedad de platanera Gruesa, selección local de Dwarf Cavendish, ha experimentado un importante aumento en los últimos años en las Islas Canarias, tanto al aire libre como bajo invernadero. La eliminación de hojas, tras la floración, es una práctica habitual en los cultivos bajo invernadero. Asimismo es frecuente la pérdida de hojas por el efecto de los vientos en los cultivos al aire libre. El objetivo de este trabajo es evaluar, mediante simulación de pérdida foliar por daños mecánicos, la influencia que tiene la disminución de superficie foliar sobre el llenado y cosecha de la fruta en dicho cultivar. Para ello, cuatro meses antes de la cosecha se efectuaron cinco niveles de defoliación: $0 \%, 25 \%$, $50 \%, 75 \%$ y $100 \%$. Se valoran dos métodos diferentes de defoliación, eliminación de limbo foliar y tronchado de hojas con posterior corte de éstas. Se analizan y presentan datos morfológicos, fenológicos y productivos, así como valoración de la metodología empleada en este trabajo para la simulación de daños. A partir de un $25 \%$ de defoliado, equivalente a 7.5 hojas funcionales por planta, se detectaron diferencias significativas con las plantas testigos.

Palabras clave: Banana, técnicas culturales, daños por viento, producción, subtrópico.

\section{INFLUENCE OF LEAF REMOVAL IN YIELD OF CV. GRUESA, Musa acuminata COLLA (AAA), CULTIVATED UNDER GREENHOUSE IN THE CANARY ISLANDS}

\begin{abstract}
The banana cultivar Gruesa, a local Dwarf Cavendish selection, is increasingly planted in the Canary Islands, both in greenhouse and in the open air. Both methods present some degree of leaf loss throughout the crop cycle: the leaves of greenhouse plants are traditionally cut back after flowering, and open air plantations experience frequent wind damage. A trial was set up four months prior to harvest to evaluate fruit filling rate and yield of Gruesa subjected to five levels of defoliation $(0 \%, 25 \%, 50 \%, 75 \%$, and $100 \%$ loss of leaf area). Two defoliation techniques were used to simulate damage: removal of the midrib, and slashing the leaf blade followed later by complete removal. Morphological, phenological and production data are presented.
\end{abstract}

Index terms: banana, cultural techniques, wind damage, subtropics, production.

\footnotetext{
'(Trabalho 251-09). Recebido em: 03-11-2009. Aceito para publicação em: 15-04-2010.

${ }^{2}$ Ingeniero Técnico Agrícola, Depto. de Fruticultura Tropical, Instituto Canario de Investigaciones Agrarias, España. e mail : jcabrera@icia.es

${ }^{3}$ Ingeniero Agrónomo, Depto. de Fruticultura Tropical, Instituto Canario de Investigaciones Agrarias, Espana - email. itziarrh76@hotmail.com ${ }^{4}$ Dr. Ingeniero Agrónomo- Departamento de Fruticultura Tropical, Instituto Canario de Investigaciones Agrarias, España. e mail: vgalan@icia.es
} 


\section{INTRODUCCIÓN}

La influencia de la pérdida foliar durante el desarrollo de la inflorescencia en la producción de la platanera, ha sido estudiada por diferentes autores y bajo distintas condiciones agroclimáticas, Rodrigo Gómez (1990), Robinson et al.(1992), Eckstein et al. (1996), Cayon (2001).

En las zonas productoras del trópico donde las plantaciones sufren importantes daños por ataques del hongo Mycosphaerella musicola, sigatoka, el deshoje es practicado como medida profiláctica. En el cultivo bajo invernadero en las Islas Canarias, aunque no hay ataques de sigatoka, al no producirse desgarros de hojas por el viento, es práctica habitual eliminar hojas manualmente después de producirse la emisión del racimo (GALÁN SAÚCO, 1992). En los cultivos al aire libre de las islas se debe actuar con precaución, sobre todo en determinadas épocas del año en que el viento puede producir una importante pérdida de superficie foliar en las plantas comprometiendo la cosecha. El conocimiento del número mínimo de hojas sanas que la planta debería mantener, una vez emitido el racimo, puede permitir programar correctamente el deshoje como práctica cultural sin afectar al correcto desarrollo del racimo y estimar la pérdida de producción en el caso de disminución de la superficie foliar por viento o granizo durante la fase de fructificación. Este dato es de sumo interés en el Seguro Colectivo del Plátano de Canarias, el cual tiene suscrito la práctica totalidad de los productores de las Islas. El objetivo de este trabajo es estudiar la influencia de la disminución de superficie foliar después de la floración sobre la producción.

\section{MATERIAL Y MÉTODOS}

Los ensayos se efectuaron bajo invernadero en la finca La Laja, Buenavista, Tenerife, Islas Canarias. $28^{\circ}, 23^{\prime}$ latitud Norte a $40 \mathrm{~m}$ de altitud. Para llevar a cabo este ensayo se utilizaron plantas adultas en fase productiva del cultivar Gruesa del subgrupo Cavendish de Musa acuminata Colla (CABRERA CABRERA; GALÁN SAÚCO, 2006), con fecha media de emergencia del racimo el 30 de Septiembre del 2004, eligiéndose aquellos que presentaban mayor homogeneidad en cuanto a su estado fenológico. En el mes de Octubre de 2004 se eligieron las plantas a utilizar en el ensayo, marcando al azar 12 plantas para cada tratamiento, en total 120 plantas.

La defoliación del cultivo fue realizada los días 11 y 12 de Noviembre de 2004, empleando dos métodos distintos, ambos a su vez divididos en cuatro gradaciones diferentes:

1) Defoliación mediante tronchado de hoja.

Este método se aplicó en distintos grados a 48 plantas (12 por tratamiento) y consistió en eliminar parte de la superficie foliar de la planta con el tronchado de las hojas en el punto medio de la nervadura central ( $50 \%$ de limbo tronchado) y/o en el punto medio del pecíolo ( $100 \%$ de limbo tronchado) (Figura1). Las plantas a las que se les aplicó este tipo de defoliación las identificamos con una $\mathrm{T}$ y dependiendo de la intensidad de la defoliación, de la siguiente forma: T-25 (25\% de reducción de la superficie foliar), T-50 (50\% de reducción de la superficie foliar), T-75 (75\% de reducción de la superficie foliar), T-100 (100\% de reducción de la superficie foliar). Las defoliaciones se efectuaron de la forma que se indica en la tabla 1, donde se especifican las hojas que fueron manipuladas y en qué porcentaje.

2) Defoliación mediante rasgado y eliminación de limbo.

Este método se aplicó a otras 48 plantas (12 por tratamiento) y consistió en el rasgado y eliminación del limbo desde el ápice hasta el punto medio del limbo (50\% de limbo eliminado) y desde el ápice de la hoja hasta la base del limbo (100\% de limbo eliminado), conservando en ambos casos la nervadura central (figura1). Las plantas a las que se les aplicó este tipo de defoliación las identificamos con una $\mathrm{L}$ y dependiendo de la intensidad de la defoliación, de la siguiente forma: L-25 (25\% de reducción de la superficie foliar), L-50 (50\% de reducción de la superficie foliar), L-75 (75\% de reducción de la superficie foliar), L-100 (100\% de reducción de la superficie foliar).

En ambos métodos la defoliación se efectuó empezando por las hojas superiores, las más funcionales y expuestas a daños por viento, hasta alcanzar la Hoja X (décima hoja contada a partir de la última emitida antes de la aparición del racimo sin contar la hoja bracteal) en las defoliaciones más intensas. Las defoliaciones se efectuaron de la forma que se indica en la tabla 1 , donde se especifican las hojas que fueron manipuladas y en qué porcentaje. La figura 2 ilustra algunos ejemplos de estos tratamientos.

Previamente a todas las plantas, a excepción de las testigo denominadas Test 2 , se le dejaron solo las 10 hojas superiores, eliminándose las restantes, con el objeto de poder cuantificar eficazmente el porcentaje de defoliación a aplicar posteriormente. Asimismo, las hojas bracteales fueron eliminadas.

Se utilizaron dos tipos de plantas testigo, 
12 plantas por tipo, las denominadas Test 1, a las que se dejó las diez hojas superiores íntegras y las denominadas Test 2 en las que se mantuvo el número de hojas presentes en el momento de practicar la defoliación (entre 11 y 14).

Después de efectuados los tratamientos de defoliación se realizó un seguimiento mensual al incremento de calibre del dedo característico de la mano central del racimo, tomándose asimismo distintos parámetros morfológicos, fonológicos y productivos, mostrados en la tablas 2 y 3 .

El análisis estadístico realizado se llevó a cabo utilizando el programa estadístico Systat Main 10. Se realizó análisis de varianza, y comparación entre medias mediante el test de Tukey al $0.5 \%$

\section{RESULTADOS Y DISCUSIÓN}

Tras realizarse el análisis estadístico, se observó que no existían diferencias significativas entre el defoliado mediante eliminación de limbo (Tratamientos L) y el defoliado mediante tronchado de hoja (Tratamientos T), asimismo tampoco se observaron diferencias entre los dos tipos de testigo (Test 1 y Test 2). Por ello se agruparon los tratamientos en 5, denominados; D0 (Test 1 y Test 2), D25 (L 25 y T 25), D50 (L 50 y T 50), D75 (L 75 y T 75) y D100 (L 100 y T 100). Esto es de gran importancia para posteriores estudios, ya que se podrá elegir indistintamente el método más adecuado para cada caso, lo que facilitará considerablemente el trabajo de campo.

No se detectaron diferencias significativas respecto a los parámetros morfológicos iniciales: altura y grosor del seudotallo, $\mathrm{n}^{\mathrm{o}}$ de manos, $\mathrm{n}^{\mathrm{o}}$ de dedos de la $2^{\text {a }}$ mano superior e inferior, (Tabla 2), lo cual era de esperar ya que las plantas fueron elegidas con similares características morfológicas y fenológicas, garantizando así la homogeneidad de los tratamientos.

El incremento mensual del calibre del dedo característico de la mano central del racimo (Tabla 3) no mostró inicialmente diferencias significativas entre tratamientos, pero éstas fueron ampliándose hasta el momento de la recolección donde ya son significativas, con una relación directa entre el porcentaje de defoliación y el calibre alcanzado por el fruto. Esta disminución en el calibre de los frutos queda también reflejada en los dedos característicos de la $2^{\mathrm{a}}$ mano superior e inferior (Tabla 2) e incluso se llega a detectar alguna diferencia significativa en la longitud de los dedos. Esta progresiva pérdida de calidad observada concuerda a grandes rasgos con lo observado por Rodrigo Gómez (1990) que detecta diferencias significativas en calidad de la fruta (calibre y grosor) en los tratamientos en que se conservaban 4 hojas y a veces 6 , pero no en los casos de conservación de 8 hojas. Dichas diferencias se traducen en una disminución del peso bruto del racimo, una vez cosechado con una relación directa entre el porcentaje de defoliación y el peso bruto alcanzado por el racimo, que también disminuye. Esta merma del rendimiento de acuerdo con los trabajos de Eckstein et al., (1996) también podría explicarse por una progresiva disminución de la capacidad fotosíntética motivada por la subsiguiente pérdida de superficie foliar. A esta disminución de peso del racimo se le añade la pérdida de fruta por una maduración precoz y falta de llenado por efecto del defoliado. La pérdida de valor comercial, expresada en $\mathrm{kg}$ por disminución de peso y madurez anticipada de fruta, es directamente proporcional al porcentaje de defoliado efectuado.

E1 tratamiento D25, equivalente a haber dejado 7,5 hojas funcionales, solo mostró pequeñas diferencias con el testigo D0 pero nunca significativas, lo cual está muy próximo a las 8 hojas mencionado por Robinson et al.1992, como necesarias para evitar pérdidas de cosecha. 
TABLA 1 - Porcentajes de defoliación aplicados (D \%) y hojas manipuladas. Hoja I = última hoja emitida tras la hoja bracteal.

\begin{tabular}{ccccccccccc}
\hline D \% & Hoja I & Hoja II & Hoja III & Hoja IV & Hoja V & Hoja VI & Hoja VII & Hoja VIII & Hoja IX Hoja X \\
\hline D25 & 100 & 50 & 50 & 50 & 0 & 0 & 0 & 0 & 0 & 0 \\
D50 & 100 & 100 & 50 & 50 & 50 & 50 & 50 & 50 & 0 & 0 \\
D75 & 100 & 100 & 100 & 100 & 100 & 50 & 50 & 50 & 50 & 50 \\
D100 & 100 & 100 & 100 & 100 & 100 & 100 & 100 & 100 & 100 & 100 \\
\hline
\end{tabular}

TABLA 2 - Datos morfológicos, fenológicos y productivos. D100 equivale a un 100\% de defoliación, D75 a un $75 \%$, D50 a un $50 \%$, D25 a un $25 \%$ y D0 son las plantas testigos.

\begin{tabular}{l} 
Seudotallo \\
\cline { 5 - 7 }
\end{tabular}

Medias en la misma columna acompañadas de la misma letra no presentan diferencias significativas entre sí (P $\leq 0.05)$ según el test de Tukey) 


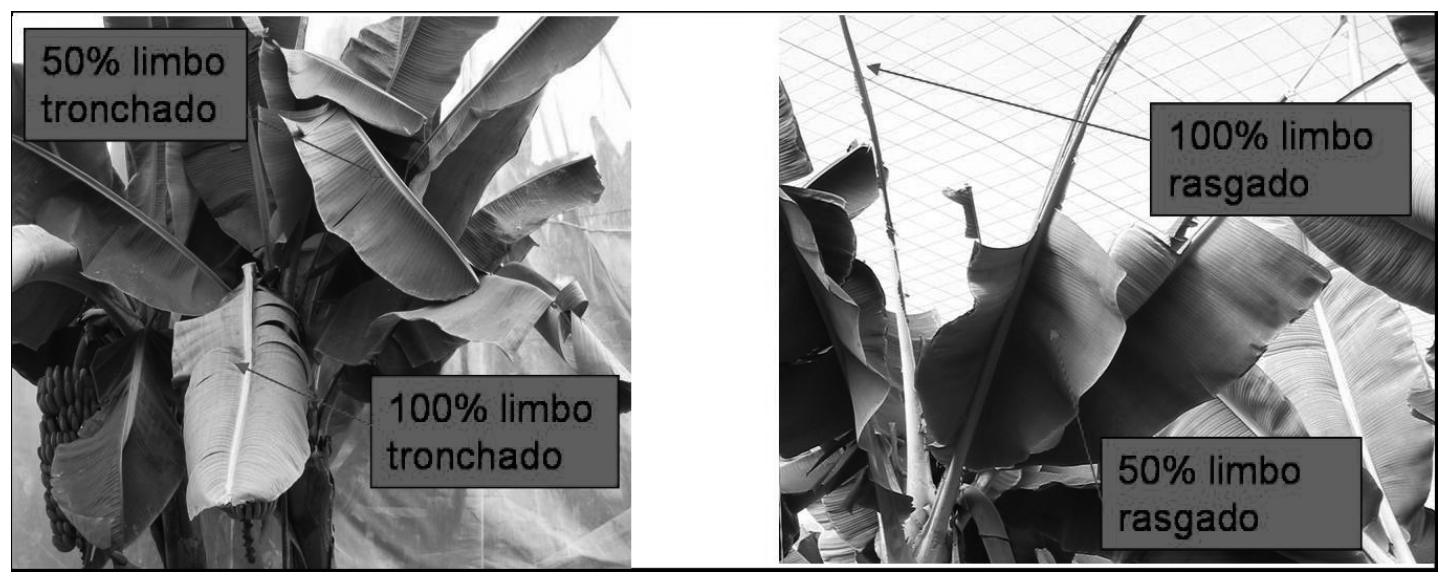

FIGURA 1- Métodos de defoliación empleados en el ensayo (tronchado y rasgado).

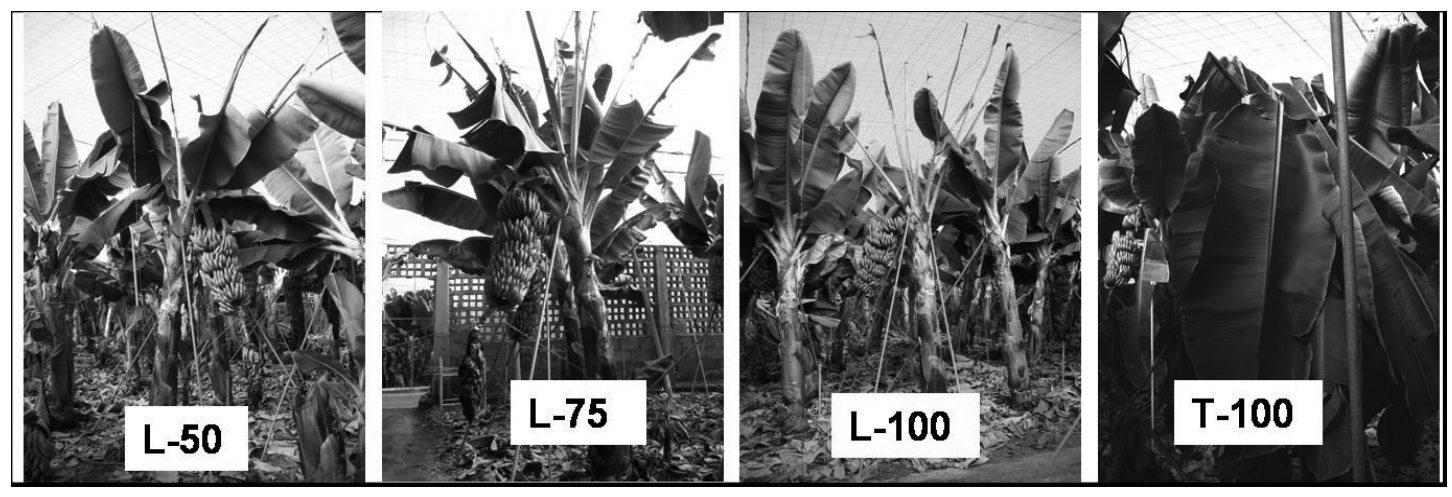

FIGURA 2- Ejemplos de tratamientos de defoliación aplicados.

\section{CONCLUSIONES}

En las condiciones del ensayo efectuado se pone de manifiesto que:

1-Los dos métodos de defoliado practicados, "rasgado y eliminación de limbo" y "tronchado" no presentaron diferencias entre ellos en cuanto a los parámetros estudiados.

2-El incremento del calibre del dedo característico de la mano central es inversamente proporcional al porcentaje de defoliado efectuado.

3-El peso bruto del racimo es inversamente proporcional al porcentaje de defoliado efectuado tras las floración.

4-La pérdida de valor comercial, expresada en $\mathrm{kg}$, por disminución de peso y madurez anticipada de fruta, es directamente proporcional al porcentaje de defoliado efectuado tras las floración.

\section{AGRADECIMIENTOS}

A los Hnos. Acevedo González, Finca La Laja, Buenavista, Tenerife, por su inestimable apoyo para la realización de este trabajo así como al Ingeniero Técnico Agrícola Juan Carlos Hernández Conde, responsable técnico de la explotación, sin cuya colaboración no hubiera sido posible llevarlo a buen término. A la Agrupación Española de los Seguros Agrarios Combinados, AGROSEGURO, por el apoyo técnico y económico prestado. 


\section{REFERÊNCIAS}

CABRERA CABRERA J.; GALÁN SAÚCO, V. Evaluation of the banana cultivars Zelig, Grande Naine and Gruesa under different environmental conditions in the Canary Islands. Fruits, Paris, v. 60, n. 6, p.1-13, 2006.

CAYON S, G. "The evolution of photosynthesis, transpiration and chlorophyll during the development of leaves of plantain (Musa AAB Simmonds)". Infomusa, Montpellier, v.10, n.1, p.12-15, 2001.

ECKSTEIN, K; ROBINSON, J. C.; FRASER, C. "Physiological responses of banana (Musa AAA; Cavendish sub- group) in the subtropics. V. Influence of leaf tearing on assimilation potential and yield". Journal of Horticultural Science, Asford, v.71, n. 2, p. 503-514, 1996.
GALÁN SAÚCO, V. Los frutales tropicales en los subtrópicos: II. el plátano (Banano). Madrid: Ed. Mundi Prensa, 1992. 177 pp.

ROBINSON, J. C.; ANDERSON, T.; ECKSTEIN, $\mathrm{K}$. The influence of functional leaf removal at flower emergence on components of yield and photosynthetic compensation in banana. Journal of Horticultural Science, Asford, v. 67, n. 3, p. 403-410, 1992.

RODRIGO GÓMEZ, P. Efecto del corte de hojas sobre la producción y desarrollo en invernadero de la platanera (Musa Acuminata colla (AAA) cv. Pequeña enana). 1990. 92f. Monografia (Trabajo Fin de Carrera) - Escuela Técnica Superior de Ingenieros Agrónomos. Universidad de Córdoba, Córdoba, 1990. 